\title{
Le Haut Dadès: Un Site Montagnard De Grande Valeur Touristique
}

\author{
Mustapha Aafir \\ Laboratoire de Recherche : \\ Dynamique, Espace, Patrimoine et Développement Durable \\ Faculté Polydisciplinaire de Taza. \\ Université Sidi Mohammed Ben Abdellah.Fès, Maroc \\ Aboubakr Sabiri \\ Laboratoire d'Analyses Géo-Environnements \\ et d'Aménagement, et Développement Durable \\ Université Sidi Mohammed Ben Abdellah, Fès, Maroc
}

Doi:10.19044/esj.2018.v14n17p88 URL:http://dx.doi.org/10.19044/esj.2018.v14n17p88

\begin{abstract}
Absract
Dades is a unique valley, defined by a long green oasis covering the extended banks of Dades river with variety of trees that embellish and decorate the oasis excluding palm trees. Palm trees are not cultivated there as the weather is quite cold especially in winter. Dades is a melting pot in which variety of high-quality touristic attractions mingled and blended together to create irresistible glamor that pulls its visitors to fell in love with the area. These touristic attractions create fairly considerable natural and cultural heritage. Accordingly, the natural environment is well diversified (gorges, high mountains, sources, etc.), whilst, the cultural heritage is well built upon significant authentic cultural legacy (kasbahs, folklore, festivals, the religious cults and buildings, handicrafts and gastronomy).

Indeed, tourism is the dynamic sector in the area. Since it benefits from various assets taking in account the richness of the cultural and natural heritage of this region, including the attractiveness of the architectural heritage, the specificity of the folklore, the finesse of the gastronomy, crafts, historic sites, and the diversity of the natural landscapes. All these potentials that are, in fact, a valuable heritage, must be integrated into the development strategy of the touristic products of DadesValley to reinforce its originality and enhance its attractiveness. In short, this article is a sort of a presentation of these characteristics with much focus on the general aspect of tourism in Dades and the useful elements for the development of a touristic mountainous area.
\end{abstract}


Keywords: Mountain, Assets, Tourism, Dades, Morocco

\section{Résumé}

Le haut Dadès est une vallée caractéristique car elle ne dispose pas de palmier dattier, mais elle abrite un ensemble d'atouts touristiques de haute qualité. Ils sont d'ordre naturel, culturel et patrimonial, et qui doivent être des éléments d'une configuration touristique originale. Le contexte naturel est bien diversifié (gorges, hautes montagnes, sources, etc.), et le patrimoine construit est d'une valeur culturelle considérable (kasbahs, folklores, festivals, les cultes et édifices religieux, l'artisanat et la gastronomie). Le mode de vie des habitants et les pratiques quotidiennes adaptées au climat local.

Alors, dans un contexte assez difficile, comme dans les montagnes marocaines, toutes les ressources territoriales doivent être mises au service du développement. Le tourisme est l'un des secteurs porteurs. Il présente des atouts divers vu la richesse du patrimoine culturel et naturel de ces espaces, l'attractivité de leur patrimoine architectural, la spécificité de leurs folklores, la finesse de leur gastronomie, leur artisanat, les sites historiques qu'elles abritent, et la diversité de leur paysage naturel. Ce sont autant de potentialités qui représentent en fait un patrimoine de grande valeur, et qui doivent être intégrés dans la stratégie de développement du produit touristique de haute vallée du Dadès, car ils renforcent son originalité et accentuent son pouvoir attractif. Cet article présente ces caractéristiques et met l'accent sur l'aspect général de tourisme au Dadès et les différents éléments utiles en matière de développement d'un site touristique montagnard.

Mots-clés : Montagne, Atouts, Tourisme, Dadès, Maroc

\section{Introduction}

$\mathrm{Au}$ cours des trois dernières décennies, plusieurs actions pour le développement du tourisme rural ont été réalisées dans ce domaine. Ces réalisations peuvent être des références significatives pour l'évaluation des impacts de l'investissement touristique dans ce domaine fragile. Il est donc temps d'évaluer les impacts socio-économiques, spatiaux et environnementaux des actions entreprises, trois décennies après leur mise en œuvre, et se poser la question de base sur la durabilité du développement induit et la pertinence de certains choix retenus.

Dans un contexte assez difficile comme dans les vallées au sud-est marocain, toutes les ressources du milieu doivent être mises au service du développement. Le tourisme culturel est dans ce cas l'un des piliers potentiels de ce développement local. En plus de la richesse de la vallée du Dadès en éléments attractifs comme le patrimoine architectural, la spécificité 
$\mathrm{du}$ folklore, la gastronomie, l'artisanat et les sites historiques, leur base économique fondamentale est pourtant l'agriculture. Celle - ci est toujours irriguée suivant des systèmes traditionnels et se pratique au fond des vallées et sur des terrasses bien aménagées, qui représentent en fait un patrimoine de grande valeur. Ce patrimoine hydro- agricole doit s'intégrer dans la stratégie de développement du produit touristique montagnard car il renforce son originalité et accentue son pouvoir attractif.

\section{- Aperçu général sur le tourisme rural au Dadès}

Les montagnes ont longtemps été considérées comme des territoires inconnus, lointains et difficiles à exploiter et effrayants. Mais elles peuvent devenir, grâce à leurs atouts patrimoniaux, culturels et environnementales, des espaces attractifs où le tourisme et les loisirs peuvent transformer les handicaps d'autrefois (paysages, froid, neige, soleil, contrastes orographiques, etc.) en formidables atouts de développement (AAFIR, 2016).

Ainsi le tourisme peut renouveler l'histoire des pratiques montagnardes et des paysages par des fonctionnalités modernes adaptées, à la fois conservatrices de ces héritages et rentables. Le tourisme rural et de montagne et l'aménagement de la montagne à ces fins est une nouvelle orientation engagée en Europe après la seconde guerre mondiale (MESPLIER et BLOC-DURAFFOUR, 1997). Au Maroc, le développement du tourisme depuis le début des années soixante-dix profita d'une façon timide aux zones rurales et de montagne. Mais l'approche alors adoptée même en milieu rural, visait la création de grandes unités d'accueil. La zone d'étude profita alors de la construction d'un hôtel classé au centre chef-lieu de la commune rurale de Boumalne du Dadès. Mais avant d'aborder le sujet, nous présentons d'abord les potentialités et réalisations en mettant l'accent sur l'analyse des deux territoires ou cette activité a été considérable. Il s'agit de la municipalité de Boumalne Dadès et de la commune d'Aït Sedrat Jabalia Soufla (Figure $\mathrm{n}^{\circ} 1$ ). Mais avant d'aborder le sujet, nous présentons d'abord les potentialités et réalisations en mettant l'accent sur l'analyse des deux territoires ou cette activité a été considérable. Il s'agit de la municipalité de Boumalne Dadès et de la commune d'Aït Sedrat Jabalia Soufla (Figure $\mathrm{n}^{\circ} 1$ ). 


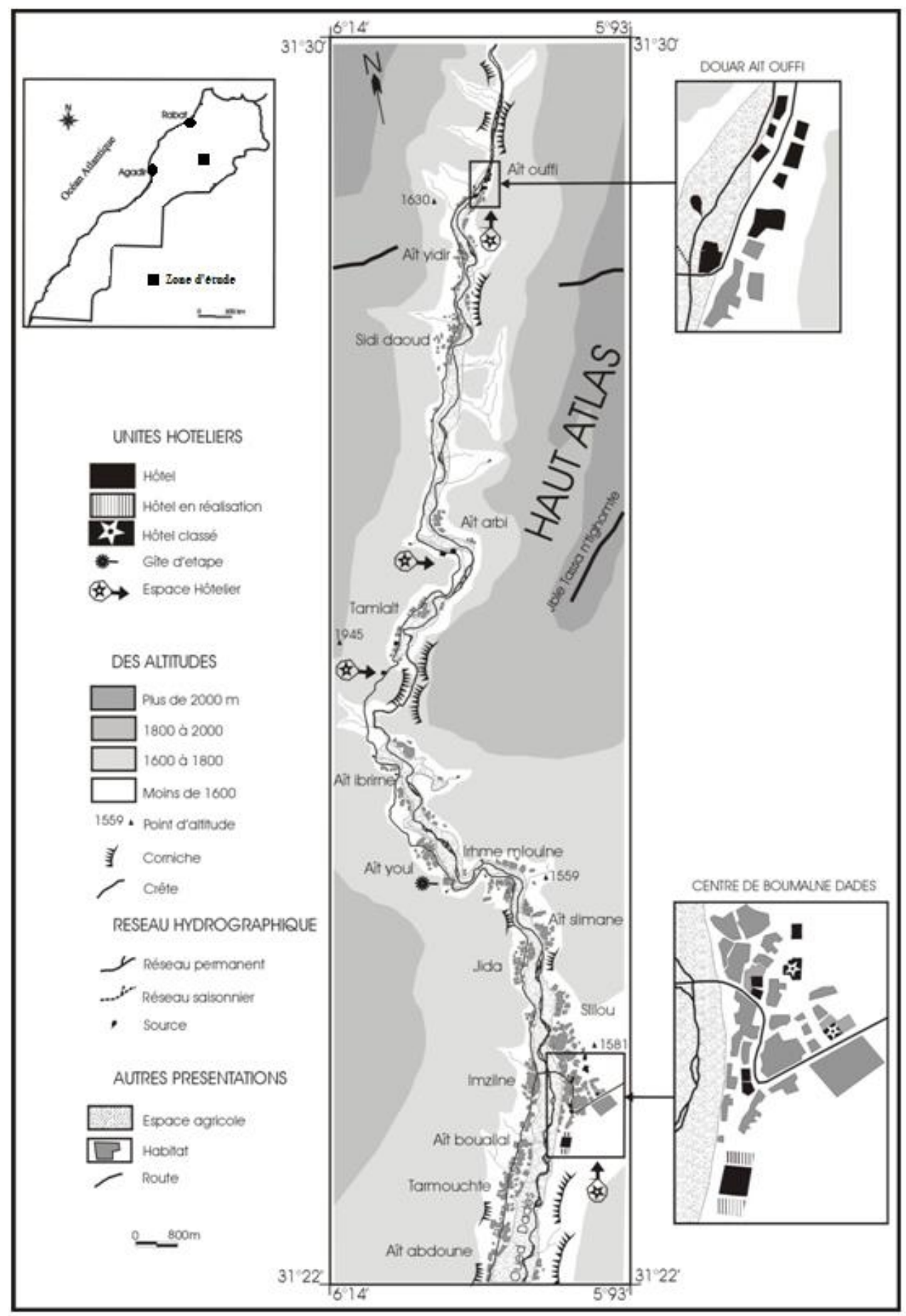

Figure $\mathrm{n}^{\circ} 1$ : Carte de potentialités touristiques de la zone étudiée 
Carte dessinée sur la base des données topographiques de la carte du périmètre du Dadès au 1/ 2.000 et des données collectées lors des enquêtes de terrain, menées 2005

Une importante infrastructure d'accueil et de services touristiques, a été réalisée au fil des années. Elle est composée de gîtes d'étape et d'un nombre important de cafés et boutiques de produits alimentaires. La figure $\mathrm{n}^{\circ} 2$ illustre une croissance rapide du nombre des hôtels dans la région, avec une capacité de 381 chambres, disposant de 771 lits. Au cours des trois dernières décennies, le nombre total d'unités d'accueil au cercle de Boumalne s'est élevé à 52 hôtels et 20 gîtes d'étapes.

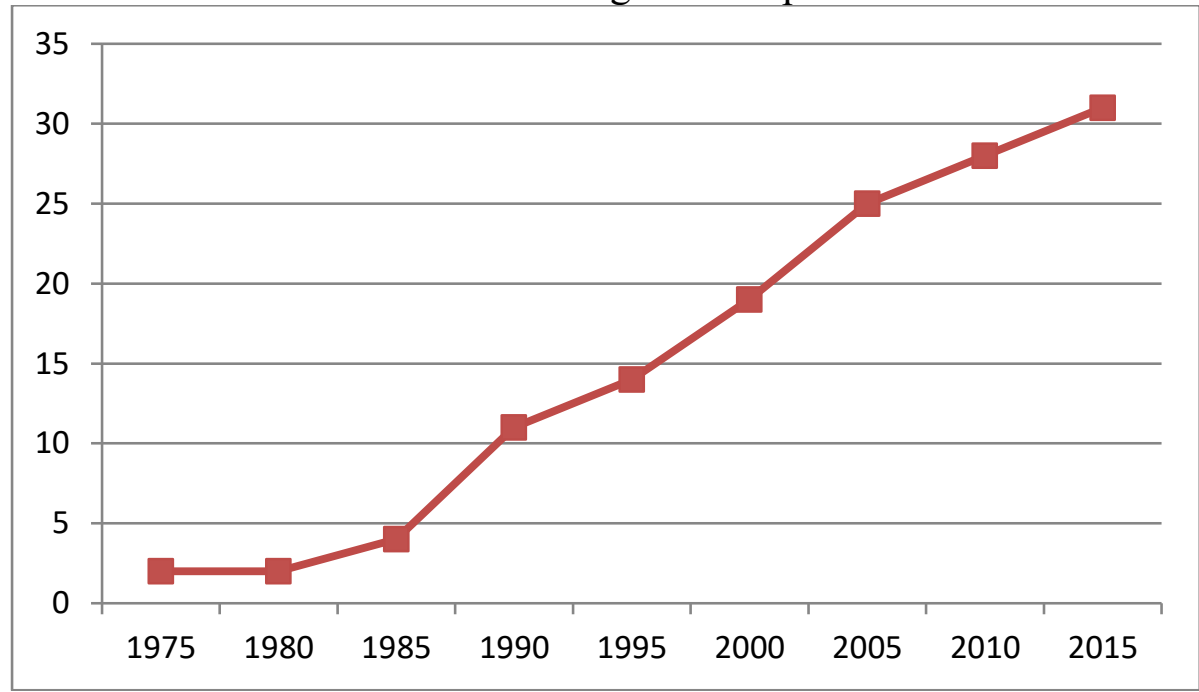

Figure $\mathrm{n}^{\circ} 2$ : Développement de nombre des hôtels dans la municipalité de Boumalne et la commune d'Aït Sedrat Jebel Soufla (1975-2015)

Source: enquête sur terrain, 2016

Le nombre des chambres connaît aussi un développement croissant au fil des années. 104 chambres ont été construites en 1972. Ce chiffre est resté stable jusqu'en 1989; année ou le nombre s'est renforcé par la construction d'un total de 42 chambres supplémentaires. Au cours des années quatre-vingt-dix, les chambres supplémentaires créées s'élève à 64 pièces. Mais la répartition spatiale de ces chambres (dont le total est de 489) diffère. La plupart des hôtels existants, ont connu une extension. $40 \%$ des unités ont été développées totalisant un ajout de chambres égal à 197 pièces (voir le tableau $\mathrm{n}^{\circ} 1$ ). 
Tableau n ${ }^{\circ}$ 1: Développement de nombre des chambres de la commune de Boumalne Dadès et Aït Sdrat Jbel Soufla depuis les années soixante-dix à 2001

\begin{tabular}{|c|c|c|c|c|c|}
\cline { 2 - 5 } \multicolumn{1}{c|}{} & $\begin{array}{c}\text { Avant } \\
1980\end{array}$ & $\begin{array}{c}1980- \\
1989\end{array}$ & $\begin{array}{c}1990- \\
1999\end{array}$ & $\begin{array}{c}2000- \\
2016\end{array}$ & Total \\
\hline Nombre des hôtels existants & 2 & 7 & 10 & 12 & 31 \\
\hline $\begin{array}{c}\text { Nombre des chambres initialement } \\
\text { construites }\end{array}$ & 104 & 42 & 86 & 60 & 292 \\
\hline Total des chambres ajoutées & 29 & 35 & 85 & 48 & 197 \\
\hline Total des chambres & 133 & 77 & 171 & 108 & 489 \\
\hline
\end{tabular}

Source : AAFIR, 2016, avec modification.

Cependant on remarque que le développement touche les établissements des années quatre-vingt-dix, plus que d'autres. De ce fait les hôtels construits durant cette période se sont élargis par la construction d'un total de 85 chambres. Par contre les hôtels crées durant les années quatrevingts n'ont connu qu'un ajout de 35 chambres, ainsi que les unités crées après 2000, s'élargis avec 48 chambres. De plus, les unités hôtelières édifiées dans les années soixante-dix ont augmenté de 29 chambres.

Le Dadès constitue donc un point d'accueil touristique principal au niveau local. En comparaison avec les vallées voisines le Dadès est moins équipé en hôtels que le Todgha (à l'est) mais mieux placé par rapport au M'Goune à l'ouest (figure $n^{\circ} 3$ ). Les hôtels existant dans la vallée du Dadès sont sur le territoire de la commune de Boumalne Dadès et Aït Sdrat Jbel Soufla avec une proportion de $71.42 \%$.

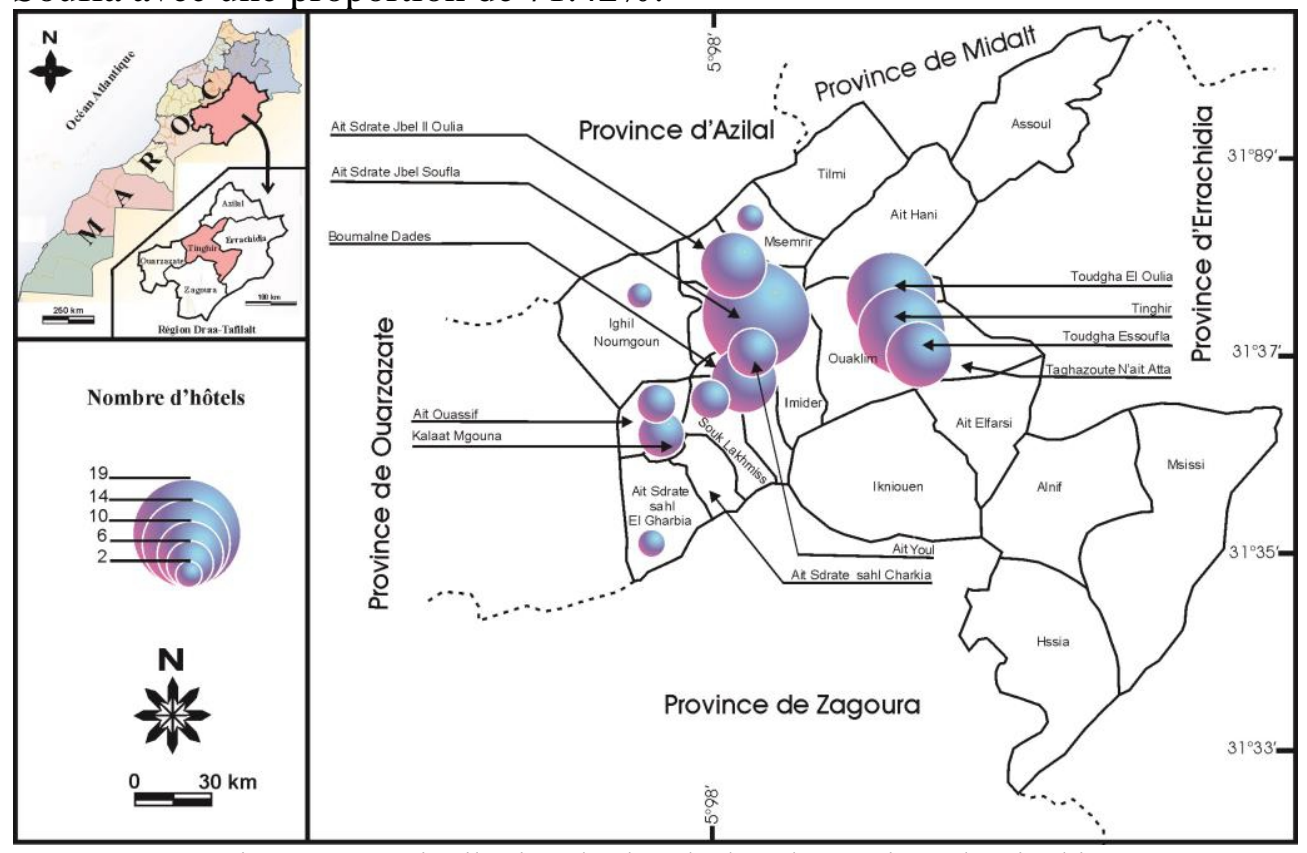

Figure $\mathrm{n}^{\circ}$ 3: Distribution des hôtels dans la province de Tinghir Source : AAFIR, 2016. 
La figure $\mathrm{n}^{\circ} 3$ illustre la distribution de ces unités hôtelières localement. Alors, au cours des quatre dernières décennies, le nombre total d'unités d'accueil s'est élevé à 112 hôtels et 48 gîtes d'étapes. La commune rurale d'Aït Sedrate Jbel Soufla vient au premier rang, avec ses 19 hôtels. En ajoutant les trois hôtels de Msemrir et les 5 autres de la commune rurale d'Aït Sedrate Jbel Soufla, la zone amont de la vallée du Dadès totalise 50 hôtels, soit 44.64\% du nombre total d'hôtels de la zone étudié.

\section{Les atouts touristiques de la zone : attractivité territoriale du Dadès}

Le Maroc possède des atouts naturels et historiques très importants et un patrimoine culturel riche et diversifié. Le tourisme montagnard au Maroc s'affirme de plus en plus comme une véritable priorité de son plan de développement, compte tenu de son impact positif économique et social. Et depuis le début de cette activité touristique les visiteurs ont découvert des espaces magnifiques. Le Dadès demeure la cité la plus originale de cette activité. Et d'ailleurs, Ce site relève des huit territoires (destinations) touristiques s'imposeront comme les vitrines du Maroc en matière de développement, dans le cadre de la stratégie de développement touristique «Vision 2020 ${ }^{9} »$, nommé Atlas et Vallées, ancré sur les vallées et les oasis, ainsi que le haut Atlas, qui se positionnera comme la destination phare de l'écotourisme et du développement durable méditerranéen (AAFIR et All 2014). Alors, d'importantes potentialités touristiques se trouvent dans l'aire d'étude. Elles sont d'ordre naturel, culturel et patrimonial par exemple.

\section{Eléments des paysages naturels}

Au départ du centre de Boumalne du Dadès en allant vers le nord, la route vers Msemrir traverse des paysages panoramiques le long de la vallée. La géologie offre des formes et formations clairement distinctes en âge, en couleur et en épaisseur. La stratigraphie montrant une alternance de couches argileuses roches rouges, parfois violacées, aux bancs calcaires jaunâtres créant un décor naturel grandiose dans ce contexte. Au fur et à mesure que la route s'élève, le ruban de la verdure centré dans la vallée se réduit pour n'être plus qu'un mince ruban d'herbe verte en amont. La vallée du Dadès a été taillée dans le calcaire globalement d'âge liasique et jurassique engendrant des belles gorges « canyon » spectaculaires aux parois vertigineuses. Ainsi, sur le plan touristique, le Dadès se caractérise par :

9 - La Vision 2020 adopte une démarche analytique, exhaustive et rigoureuse. Elle est inspirée par la volonté de passer à une démarche d'aménagement du territoire structurante en termes d'emplois et de développer la compétitivité du tourisme marocain en se basant sur les atouts des régions selon une dimension territoriale et intégrée. (www.tourisme.gov.ma). 
- la fraîcheur des oasis qui contraste avec le climat chaud, de type aride et saharien qui domine le Sud du Haute Atlas. Le climat est sec et agréable, surtout au printemps et à l'automne,

- la variété saisissante des paysages: vallées profondes, gorges, canyons vertigineux et sommets érodés. Ces éléments naturels donnent une valeur touristique typique au paysage,

- la vallée se caractérise par son étagement typique en raison d'un emboîtement de terrasses fluviatiles supportant d'importantes des activités maraîchères et des éléments d'un patrimoine écologique qu'il convient de préserver tout diversifiant ses modes d'exploitation.

\section{Les éléments du patrimoine architectural et culturel \\ - Les spécificités architecturales de l'habitat ancien}

Bien que l'habitat évolue et se modernise dans l'aire d'étude, le style architectural traditionnel persiste. La plupart des douars de la vallée montrent souvent des kasbahs typiques, coalescentes et jumelées ou individualisées et isolées. Elles s'imposent à la fois par leur localisation et leurs caractéristiques techniques et architecturales.

La localisation des Kasbahs sur des sites stratégiques, suffisamment élevés pour dominer le paysage, généralement à mi - versants de la vallée et sur les pentes et pieds de montagne (EL MANOUAR 2012), montre que cet habitat aggloméré suit une logique bien raisonnée pour servir ses objectifs fonctionnels. La protection des périmètres agricoles (terrasses limoneuses exploitables en agriculture) explique aussi l'installation de cet habitat aggloméré en bordures de la zone cultivable, sur la terrasse conglomératique consolidée du quaternaire moyen. Mais antérieurement (à l'époque du protectorat en avant), certains villages du Dadès Moyen (commune rurale de Khemis Dadès), s'implantent au sein même du périmètre irrigué, pour une meilleure proximité des jardins. La route actuellement attirante de l'habitat, n'a pas d'influence au début du siècle dernier vu l'autosuffisance économique adoptée et l'ouverture réduite de la société locale. Les villages agglomérés, généralement formés de grandes kasbahs dans cette zone sont donc de deux types : entités perchées dominant la vallée et entités localisée au milieu du secteur agricole. Mais ce dernier type, très ancien, disparaît progressivement sous l'influence de l'attractivité de la route et des risques d'inondation auxquels sont exposés les villages anciens des basses topographies.

L'architecture s'impose dans le paysage local en raison de ses apparences originales et caractéristiques. Les matériaux et les techniques de construction sont typiques que ce soit dans les maisons construites en terre (pisé et torchis) ou dans la majorité des maisons récemment construites en ciment. C'est un patrimoine jusqu'à nos jours bien préservé comme partout 
au sud oasien, mais ses défis sont énormes face aux mutations en cours. Les kasbahs forment un élément majeur de ce patrimoine historique. Souvent situées dans le noyau ancien des Douars, les kasbahs ont des dimensions variables. Leur architecture est globalement défensive, comportant quatre tours angulaires, décorées parfois de riches motifs dans leurs parties supérieures. L'activité touristique leur a permis, à travers cartes et photos, d'atteindre une renommée mondiale et la cinématographie, développée à Ouarzazate, trouve dans ce cadre des décors particulièrement recherchés.

\section{- L'animation culturelle et les coutumes : un atout pour le développement du tourisme rural}

Le Sud marocain en général et le Dadès en particulier est riche d'un patrimoine culturel d'une grande valeur artistique et d'une symbolique sociale et socio-économique très importante. La vie quotidienne traditionnelle est rythmée par la diversité des coutumes sociales : les modes de vie, les activités parallèles, le folklore, l'habillement et l'organisation sociale et spatiale sont riches et variés, témoignant de la vigueur de la culture, élément de la civilisation qui structure l'identité locale, encore très vivante dans ce domaine. Les chansons, les danses folkloriques, les jeux guerriers et chorégraphiques constituent un patrimoine populaire et un moyen de communication de la culture Amazigh régionale. Parmi les groupes folkloriques les plus connus, on trouve Ahidouss, Ahouach et Gnaoua.

Les Moussems, événement culturels annuels très anciens dans la zone, se déclinent sur plusieurs thèmes. Ils constituent occasions et lieux de rencontres et de festivités et peuvent être transformés en véritables pôles d'animation et d'attraction pour les touristes. Actuellement, le plus réputé est la fête des roses annuellement organisée à Kalaa M'gouna. D'autres moussems et fêtes sont organisées dans la région à l'extérieur de l'aire d'étude comme le Festival des fiançailles à Imilchil, la fête de Bougafer à Ikniouen, cercle de Boumalne, le moussem du tapis à Taznakhte et celui des dattes au Draa. Ce sont des activités qui, après une réorganisation potentielle peuvent participer à diversifier l'animation culturelle régionale au profit du tourisme.

Les Souks hebdomadaires que l'on retrouve dans la presque totalité des communes rurales, constituent de véritables carrefours d'échanges et de découverte des produits locaux. Outre leur ambiance colorée, ils offrent un lieu de curiosité et de découverte appréciable pour le touriste et le citoyen.

\section{- L'art et l'artisanat}

L'artisanat marocain, réputé aujourd'hui dans le monde entier par ses tapis, poteries et bijoux, puise son authenticité et conserve ses secrets dans 
les régions d'origine. Il représente un intérêt pour les curieux qui veulent le découvrir par eux-mêmes. C'est un facteur du développement du tourisme qui a d'ailleurs connu des mutations induites par le développement récent du tourisme dans la zone. Certains professionnels ont ouvert des ateliers de commerce (Bazars), soit aux centres comme Boumalne Dadès et Kalaa M'gouna, dans les douars ou sur les points de vente le long de la route (belles vues panoramiques, à côté des kasbahs et des hôtels) comme au douar Irhem Mellouln, Tamlalt, Aït Arbi, Aït Ouffi et dans les gorges du Dadès par exemple.

L'artisanat est un secteur à grands potentiels de développement local grâce aux synergies qu'il engendre avec de nombreuses activités comme les activités de service, le secteur du bâtiment et le tourisme par exemple. Un effort de conservation et de développement de l'artisanat s'impose pour préserver et améliorer la qualité des produits leur forme, leur esthétique et ergonomie.

La vallée du Dadès disposait d'un artisanat de qualité varié qui se reproduit en partie localement. Certains métiers sont disparus ou menacés de disparition comme le tissage du tapis, des Jellaba, Aznnar, Ahendir, Tahrouyte, Foulard, ou les produits de fer forgé très connus au douar Imziln, la bijouterie juive au douar Tiylite, les activités du bois, la fabrication des cerrures ou des outils agricoles (Tazzert) en bois, etc. Certains établissements tels que la coopérative des artisans et la coopérative des bijoutiers et ferrailleurs à Kelaat Mgouna espèrent redynamiser certaines de ces activités. Un club «foyer » des femmes a été créé au centre de Boumalne Dadès; pour travailler, sauvegarder et renforcer les métiers d'artisanat qui appartiennent au patrimoine de la région. La majorité de ces activités se pratique à l'intérieur des maisons (tissage de motifs particuliers qui font le tapis berbère, le forgeage, la décoration, architecture et techniques de construction et sculpteurs sur les murs, horticulture, etc). Au-delà la population du Dadès produit des dizaines éléments artistiques:

$\sqrt{ }$ Les meubles de maison comme les plateaux et pots de cuivre au douar Azlag, et Tahitourte (peau d'animaux), les peaux d'animaux utilisées pour traiter le lait et beurre (Tigaouite), les harnais à Aït Hdiddou, le Hanbal à Slillou, des cordes, des selles (Tabarda), des coffres de bois décorés. Le Ahndir de Aït Hdiddouet le Ahndir de Dadès.

$\sqrt{ }$ Pour les hommes, on cite la fabrication des poignard (Touzzalt) et les sacs tradtionnels "Akrabe" (sorte de trousse) ainsi que les Burnous à Aït Hdiddou.

$\sqrt{ }$ Pour les bijoux des femmes comme la fabrication des fibules «Takhllalt» et les colliers berbères (Tizerouine), boite à colle, la dot de mariage (Dablige), ainsi les habilles des femmes tels que 
Laktibe (sorte de foulard) et le foulard du gros noir (Assdouye), et les ceintures berbères (Tissouma et Tassmmarte) sont autant de produits qui font de l'artisanat local un créneau potentiel du développement local.

L'originalité du produit local est antérieurement confirmée mais elle risque d'être atteinte par les évolutions en cours, notamment en termes de design et de couleur. Des métiers d'artisanat risquent de disparaître si des mesures de réhabilitation ne sont pas adoptées. La mise à niveau de ce secteur est une mesure parallèle qu'il revient aux pouvoirs publics de mener pour soutenir le développement durable de la zone, participer à l'amélioration des conditions de vie des habitants appuyer développement prévu du tourisme.

\section{Le patrimoine agricole : un atout touristique encore inexploité}

Le «patrimoine » au sens où on l'entend aujourd'hui dans le langage officiel et dans l'usage commun, est une notion toute récente, qui couvre de façon nécessairement vague tous les biens et «trésors » hérités du passé. En effet, cette notion recouvre un certain nombre de couches superposées au fil des années, qu'il peut être utile de distinguer, car elle intervient au terme d'une longue et chaotique histoire du domaine (BABELON et CHASTEL, 1994). La diversité des patrimoines et des éléments constitutifs de chaque patrimoine, rend son analyse assez complexe. Elle l'est encore plus lorsqu'on s'intéresse à des phénomènes du patrimoine qui combinent des composantes physiques, écologiques et culturelles comme par exemple « le patrimoine hydro - agricole » formé à la fois des terrasses jardins, des techniques et des pratiques d'exploitation ancestrales (AAFIR, 2011). Nous abordons ce patrimoine dans une perspective orientée pour le soutien du développement du tourisme en approfondissant la réflexion sur les moyens de diversifier l'offre du produit touristique en intégrant les éléments de ce patrimoine hydro- agricole.

Bien que l'agrotourisme ou le tourisme à la ferme soit une pratique touristique encouragée depuis fort longtemps en milieu rural, l'offre agrotouristique apparaît relativement floue (PASCALE, 2006). Alors, ici en parle de «l'agrotourisme » qui considéré comme l'une des activités exercées à la ferme par les exploitants agricoles ou par les membres de l'exploitation, ayant un certain contenu. C'est-à-dire toutes les activités correspondant à la maîtrise et à l'exploitation d'un cycle biologique, végétal ou animal et constituant une ou plusieurs étapes nécessaires au déroulement de ce cycle, ainsi que les activités exercées par un exploitant, qui sont dans le prolongement de l'acte de production ou qui ont pour support l'exploitation... Les acteurs de l'agrotourisme, composante du tourisme vert, 
sont des exploitants agricoles qui proposent sur leur exploitation soit une activité de restauration, soit une activité d'hébergement, soit une activité de loisirs et, maintenant, une activité de vente directe de leurs produits. C'est donc l'ensemble des acteurs, qu'ils soient publics, privés ou associatifs, qui proposent des activités d'hébergement, de restauration et de loisirs en espace rural (BENTALEB et BOUAOUINATE, 2018).

$\mathrm{Au}$ Dadès, l'exploitation et l'aménagement des terrasses agricoles et une tradition très ancienne. Elles s'implantent sur des topographies étagées de dépôts fluviatiles et épandages d'âges quaternaires. Joly (1962) et SAAIDI (1987) ont distingué plusieurs niveaux quaternaires dans ces terrasses :

- $\quad$ Niveaux de piémont sud atlasique : ceux du seuil d'Imiter $(20 \mathrm{~km}$ à l'est du Dadès). C'est une série de cônes de déjections et de glacis emboîtés, dominant un complexe de niveaux récents qui émergent et se croisent vers l'aval.

Niveaux du Haut-Atlas : emboîtement des formes quaternaires en moyenne et haute montagne.

Les terrasses des moyennes montagnes des vallées du versant sud du Haut-Atlas se composent pour partie de secteurs longitudinaux, parfois élargies et souvent très étroits ou en véritables gorges (exemple de Tarhia n'Aït Hammou à Dadès). L'étagement quaternaire est souvent bien développé là où les vallées sont plus larges. Elles sont facilement reconnaissables grâce à des dépôts caractéristiques. Elles deviennent fragmentaires, moins vigoureuses, rares et discontinues dans les vallées plus étroites. En haute montagne, au-dessus de 2500-3000 m, les formes et dépôts changent de caractères en raison de l'intervention du gel et de la neige qui ont joué, et jouent encore, un rôle fondamental dans le façonnement de leur matériel.

Plus en aval, à la sortie de la montagne, ces terrasses agricoles, engendrent le contraste entre le chapelet verdoyant que forment les vallées et les immenses espaces voisins dénudés. C'est ce contraste qui détermine la valeur économique et fonctionnelle de chaque espace. Mais c'est aussi ce qui donne incontestablement à l'eau et à l'irrigation sa juste valeur patrimoniale dans ces régions arides (AIT HAMZA, 1999).

Le patrimoine hydro- agricole est donc un patrimoine riche de ses composantes physiques et écologiques et diversifié par les modes de son exploitation, les techniques et les pratiques de son entretien et les systèmes traditionnels qui l'organisent. C'est un facteur indéniable d'attractivité touristique car il renforce l'identité et l'originalité des paysages (AKDIM , 2012). 


\section{La position de transition entre le Haut et l'Anti-Atlas diversifie les paysages au Dadès}

Ces deux chaînes sont structurellement différentes et leur contraste avec 1'espace désertique bordier est encore plus grand. Cette diversité des paysages physiques est renforcée par la diversité du contexte humain, économique et urbanistique. Des villes moyenne du sud-est marocain cadrent le territoire : Ouarzazate et Errachidia et rapprochent les services. Ce qui facilite depuis plusieurs années, le développement d'un pôle touristique local axé sur l'accueil centré à Ouarzazate, Errachidia et les centres urbains les plus importants. Les opportunités du développement du tourisme rural et oasien existent mais encore peu exploitées.

\section{Les étapes du développement touristique dans la région}

Depuis les années soixante le Maroc s'appuie sur le développement $\mathrm{du}$ domaine touristique. Dans ce sens l'Etat a lancé un nombre de programmes de promotion et lois. Le Dahir du 16 juin 1971 a pour objectif de faire évoluer le tourisme au niveau de différentes régions économiques du Maroc, par la création de comités régionaux du tourisme (BAKKALI, 1974). Les zones montagnardes et oasiennes sont concernées.

Ainsi, la première unité touristique a été édifiée par l'Etat au centre de Boumalne Dadès en 1972, avec une capacité de 100 chambres et 206 lits. Une autre unité a été créée par un investisseur privé dans la commune d'Aït Sdrat Jebl Soufla, avec une capacité de 4 chambres et 10 lits. Après cette date la construction a été interrompue jusqu'en 1984. Cela s'explique par:

- La capacité hôtelière existante a été suffisante pour l'accueil de touristes encore rares.

- La stagnation du tourisme à cette époque, car les mesures de promotions sont à leur début.

Mais à partir des années quatre-vingt-dix, le développement des hôtels s'accroît. Le nombre d'unités d'accueil construites avant les années soixante-dix ne dépasse pas $10.52 \%$ et $36.84 \%$ dans les années quatre-vingt. Il atteint 52.64\% durant les années de quatre-vingt-dix (figure $n^{\circ} 4$ ). 


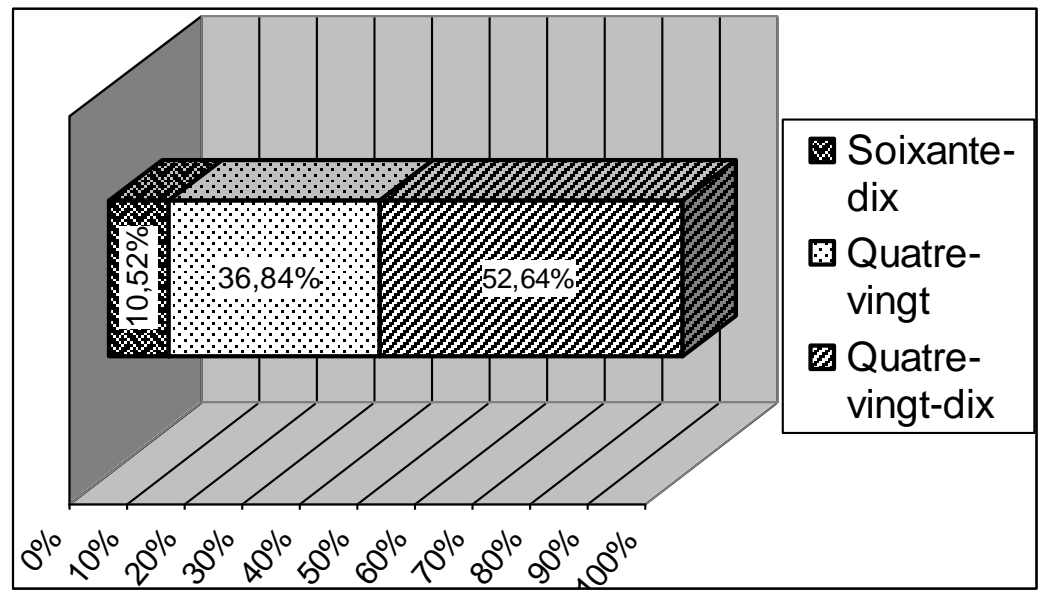

Figure $n^{\circ}$ 4: taux de développement des hôtels dans le Dadès Source: AAFIR M., 2006

Comme précédemment discuté, il est donc clair que l'infrastructure d'accueil touristique au Dadès se caractérise par l'existence de grandes unités d'accueil et d'un déclenchement du processus de construction d'hôtels et gîtes même en milieu rural. Les effets environnementaux de ces développements risquent d'être coûteux, particulièrement au niveau des ressources hydriques. Etant donné que l'espace rural est caractérisé par l'absence de réseau d'assainissement liquide et parfois du réseau de distribution de l'eau potable, chaque unité hôtelière s'équipe en eau potable et adopte son propre approche d'évacuation les déchets liquide et solides. Ce qui a des impacts négatifs en termes de mutations spatiales. Vu que les écosystèmes de montagne sont très complexes et fragiles. Leur aménagement doit prendre en compte, non seulement, les contraintes naturelles, mais aussi les imbrications entre l'homme et son environnement socio- culturel et économique (AIT HAMZA, 2000).

\section{Conclusion}

Le patrimoine culturel et naturel local est très riche et diversifié mais peu valorisé pour le développement du tourisme: paysages captivants, folklore varié, monuments historiques, agriculture irriguée sur parcelles aménagées, pastoralisme, architecture de terre, patrimoine immatériel, etc. Ce patrimoine peut servir de base pour redynamiser l'économie locale et supporter son développement durable. Des potentialités touristiques restent en friche et peuvent être développées et exploitées. Cet important héritage historique, culturel et architectural peut être un atout pour la zone si on l'intègre dans de nouvelles conceptions de développement, notamment touristique en créant l'infrastructure nécessaire mais adaptée au contexte oasien aride. 
D'un autre côté, l'éco- tourisme est un moyen de valorisation et de conservation des sites naturels et de la biodiversité sans compter son impact sur l'économie locale. De son côté, le département des Eaux et Forêts en collaboration avec PNUD, FEM et MADRPEM ${ }^{10}$ ont élaborés un projet de conservation de la biodiversité par la transhumance dans le versant sud du Haut Atlas (photo ${ }^{\circ} 1$ ).

Actuellement le tourisme demeure l'un des outils privilégiés des politiques d'aménagement et le développement durable du territoire. Le tourisme peut être programmé dans ce sens pour les montagnes, surtout que celles-ci sont devenues une des principales destinations touristiques du monde. Les visiteurs sont attirés par la beauté de ses environnements, la vaste gamme de loisirs disponibles et les possibilités de se plonger dans le patrimoine culturel, les coutumes, les arts, les traditions culinaires et l'artisanat des communautés qui y vivent. Les recettes tirées du tourisme sont devenues une source principale de revenus pour de nombreuses communautés montagnardes.

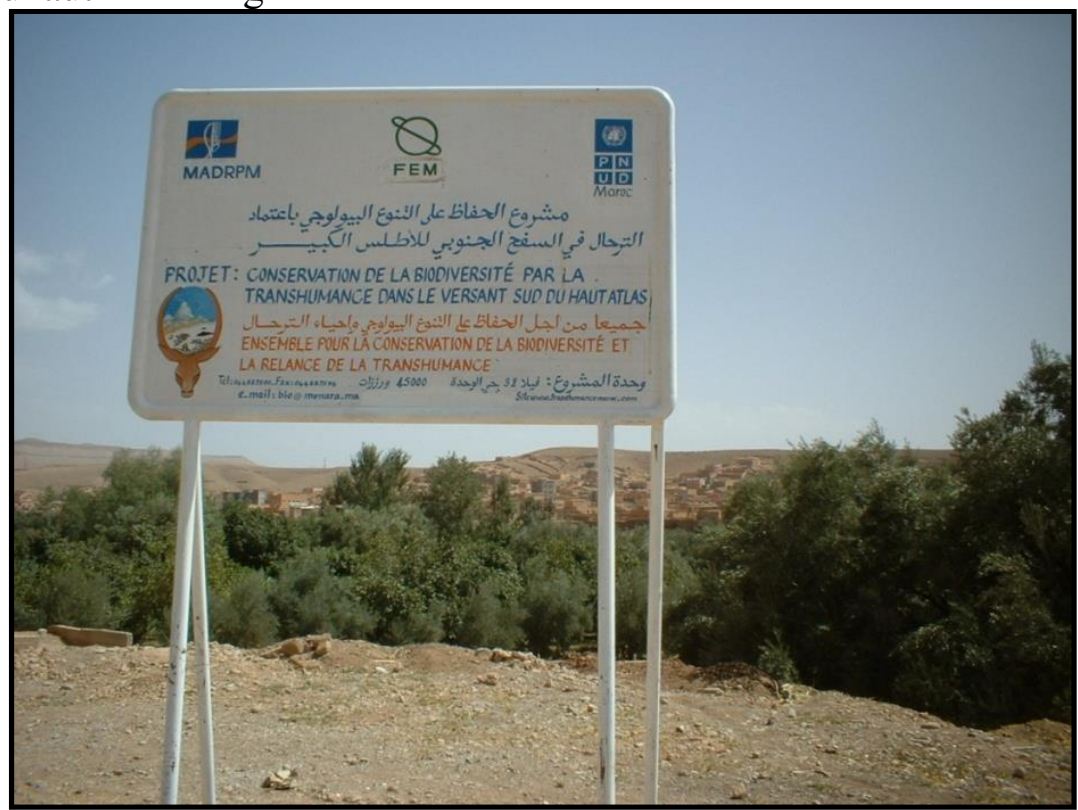

Photo $\mathrm{n}^{\circ} 1$ : Présentation du projet de conservation de la biodiversité par la transhumance dans le versant sud du Haut Atlas

Photo prise en été 2005 au douar Imzilne à la municipalité du Boumalne Dadès

${ }^{10} \mathrm{PNUD}=$ Programme des Nations unies pour le développement

FEM= Le Fonds pour l'Environnement Mondial.

MADRPM= Ministère de l'Agriculture, du Développement Rural et de Pêche Maritime. 


\section{References:}

1. AAFIR M, (2006). Les contraintes hydrologiques de l'aménagement $\mathrm{du}$ bassin versant du Dades : une approche géopolitique du développement durable. Thèse de Doctorat. Université Sidi Mohamed Ben Abdellah - Faculté des Lettres et Sciences Humaines Saïs - Fès et Université Paul Verlaine, Metz.

2. AAFIR M, (2011). Le potentiel de l'exploitation touristique des paysages agricoles en milieu oasien: cas du Dadès. In "Les produits agricoles touristiques et développement local", Édi: Association Nationale des Géographes marocains et Union Géographe Internationale, Cordonnée par Moussa Kerzazi et autres,(pp387-400). Imp: Best Inpremerie, Casablanca.

3. AAFIR M, (2016). Identification des potentialités touristiques et perspectives du développement durable: cas du Dadès (Région DraaTafilalt). Revue «Oasis du Maroc: Espace - Homme Développement durable» numéro 4. (Pp 19-42). Centre Tarik Ibn Zyad, Errachidia.

4. AAFIR M, (2016). La géopolitique de l'aménagement de l'eau à Dadès, Maroc. Editions universitaires européennes. France. (332p).https://www.editions-ue.com/catalog.

5. AAFIR M, BOUBERRIA A, et SABIRI A, BRIOUEL R, (2014). Le tourisme montagnard, une approche spatiale d'après les deux cas : Dades et Tazkka. Actes du 4ème Forum d'Ighazrane (province de Sefrou). Le tourisme de montagne, valorisation des ressources territoriales et diversifier les aspects du développement. Publication de la commune rurale d'Ighazrane. IPN. Fès (en arabe)

6. AIT HAMZA M, (1999). Mobilité socio-spatiale et développement local au sud de l'Atlas. (364p). Thèse d'Etat, Rabat,

7. AIT HAMZA M, (2000). Environnement et stratégies paysannes dans le Haut-Atlas central, in: la montagne marocaine, dynamique agraire et développement durable. Publications de la Chaire UNESCO-GAS NATURAL, FLSH; Rabat.

8. AKDIM B, (2012). Equilibre des oasis marocaine et perspectives de leur développement: approche et synthétique. Géomaghreb $\mathrm{n}^{\circ} 8$. Revue des études appliquées en sciences de la terre, géographie et aménagement. (23p). Imp-Inf-Print, Fès. (En arabe)

9. BABELON J-P. et CHASTEL A, (1994). La notion de patrimoine, édition Liana Levi. France.

10. BAKKALI M, (1974). Loi du tourisme au Maroc, étude analytique et indécis, Tome I : analyse de phénomène touristique. Société babil, Rabat. (En arabe) 
11. BENTALEB A. et BOUAOUINATE A, (2018). Valorisation touristique du patrimoine éco-culturel au Maroc méridional, cas des oasis du Draa Moyen. L'économie du patrimoine et développement durable dans les oasis et les zones vulnérable. $\mathrm{N}^{\circ}$ spéciale de la revue DIGITAR. (Pp189-201). Edi impression de 1'Université de Coimbra, Portugal.

12. EL MANOUAR M, (2012). Dads, de l'organisation sociale traditionnelle a la domination coloniale: XIXeme-XXeme siecles. Édi: Institut Royal de la Culture Amazighe, Imp: El Maarif Al Jadida, (363p). Rabat, Tome I.

13. MESPLIER Alain et BLOC-DURAFFOUR Pierre, (1997). Le tourisme dans le monde. $5^{\text {ème }}$ édition, Bréal, Rosny Cedex.

14. PASCALE M, (2006). Laurent Bourdeau and Maurice Doyon, «Agrotourisme, agritourisme et tourisme à la ferme ? Une analyse comparative », Téoros, 25-3. (Pp 59-67).

15. SAAIDI. E, (1987). Géologie du quaternaire marocaine, Smir, (pp323-331). Rabat. 